\title{
Periodontal Therapy and Low Birth Weight: Preliminary Results From an Alternative Methodologic Strategy
}

Isaac S. Gomes-Filho, ${ }^{*}$ Simone S. Cruz, ${ }^{\dagger}$ Maria da Conceiçao N. Costa, ${ }^{\dagger}$ Johelle S. Passos, ${ }^{*}$ Eneida M.M. Cerqueira, $§$ Fábio P. Sampaio, * Eliesita C. Pereira, * and Lituânia F. Miranda*

Background: The purpose of this study is to present the use of a non-randomized experimental design with multiple controls, with emphasis on a historical control group, as an alternative methodologic resource for studies on the association between periodontal disease and prematurity/low birth weight.

Methods: The sample consisted of 234 pregnant women: 54 in the Test Group (treatment of periodontal disease); 68 in Control Group I (without periodontal disease); and 112 in Control Group II (historical control group, with untreated periodontal disease). The diagnosis of periodontal disease was established by means of a complete clinical examination, using measurements of probing depth, gingival recession, clinical attachment loss, and bleeding index. The women in the Test Group were treated for periodontitis and followed-up with periodontal support therapy throughout their pregnancies. After delivery, they were reevaluated regarding their periodontal condition, and information on the newborn's birth weight was obtained. This was also done for Control Groups I and II. Descriptive analyses on the study variables were performed using the $\chi^{2}$ and Fisher exact tests. Association measurements (relative risk) were obtained using a significance level of $5 \%$.

Results: The frequency of low birth weight among the Test Group was similar to Control Group I and lower than Control Group II.

Conclusion: The results suggest that successful periodontal therapy in pregnant women suffering from periodontitis is a protective factor promoting the birth of children with normal weight. J Periodontol 2010;81: 1725-1733.

\section{KEY WORDS}

Dental scaling; epidemiology; periodontitis; pregnancy complications.

\footnotetext{
* Department of Health, Feira de Santana State University, Bahia, Brazil.

$\dagger$ Department of Epidemiology, Federal University of Vale do São Francisco, Pernambuco, Brazil.

\# Institute of Collective Health, Federal University of Bahia, Salvador, Bahia, Brazil. $\S$ Department of Biological Sciences, Feira de Santana State University.
}

$\square$ ver recent years, the significant increase in the numbers of studies evaluating the association between periodontal disease (the second most prevalent oral disease in the world) ${ }^{1}$ and prematurity/low birth weight (the most important factor relating to morbidity and mortality during early childhood) ${ }^{1}$ has consolidated the importance of this association. Both of these systemic conditions have been recognized as serious public health problems.

Most studies on this subject are observational. $^{1-4}$ Thus, no conclusions can be drawn from them regarding causality between these problems. Making a comparison with the number of observational studies that exist, only a few investigations using periodontal therapy among pregnant women are available in the literature. ${ }^{5-14}$ It is likely that the number of experimental studies on the latter topic is small because of the great complexity in conducting such studies. They demand longer duration, have high costs, and present the ethical impositions inherent to intervention studies.

Nonetheless, it is recognized that experimental study is the most appropriate type of study design for making inferences regarding the cause-effect relationship between two conditions, ${ }^{15}$ for example between therapy for periodontal disease and prematurity/low birth weight. ${ }^{11}$ The classic intervention design requires that one group receives 
treatment, while another group (also with the disease) is merely monitored without receiving this therapy. Even though the benefit from periodontal therapy for preventing undesirable gestational outcomes is unclear, ${ }^{11,16}$ some ethics committees for research on human beings (institutional review boards) have taken the view that there is a clear breach of ethical guidelines when research subjects are allocated randomly to groups with or without treatment.

Some intervention studies developed on periodontal disease and prematurity/low birth weight have shown an association, ${ }^{7,14,16-18}$ but many others have found contrary results. ${ }^{11,13,19}$ Thus, the present evidence in the literature is insufficient to be able to state categorically that periodontal infection in pregnant women is a risk factor for the birth of premature or low-weight newborns or that periodontal therapy is able to reduce the risk of these types of occurrences. ${ }^{20}$

Within this scenario, and faced with ethical issues relating to carrying out classic randomized studies, it seems opportune to put forward for debate some possible designs for intervention studies that would be conducted empirically, using alternative methodologic strategies. Examples of such strategies are seen in studies using historical control groups or external controls, ${ }^{21}$ or multiple controls. ${ }^{22}$ These alternatives may, in turn, be useful for future studies that would aim to investigate, through intervention designs, whether periodontal therapy during pregnancy might reduce the risk of the birth of premature or low-weight newborns.

Given the relevance of this topic, the few existing studies with intervention designs, and the need to generate knowledge that might consolidate this issue, the present paper had the aim of describing the preliminary results from an alternative methodologic resource: the use of a non-randomized design with multiple controls, with emphasis on the historical control group. Until now, this design has not been presented in studies evaluating the effects of periodontal therapy during pregnancy in relation to the risk of low birth weight.

\section{MATERIALS AND METHODS}

\section{Selection of Study Groups}

For this methodologic proposal, three groups of pregnant women were set up in the municipality of Feira de Santana, Bahia, Brazil: 1) Test Group, composed of women diagnosed with and treated for periodontitis during their pregnancies; 2) Control Group I, composed of women without periodontitis during their pregnancies; and 3) Control Group II, composed of women with periodontitis that were diagnosed during the immediate postpartum period.

The Test Group and Control Group I were formed by women who sought prenatal care between November
2005 and March 2007 at 12 municipal health care units in the urban zone of Feira de Santana, Bahia, Brazil. These units were selected for operational convenience, from among the 15 primary health care units and 74 family health care program units within the main urban area. The units selected were distributed in different areas of the city and they provided prenatal care that was generally destined for a clientele of low socioeconomic level (i.e., subjects who mostly had low family incomes and educational levels of $<8$ years of schooling). The Test Group $(n=54)$ was formed by women with periodontal disease who were at the outset of their pregnancies (first 16 weeks). Control Group I ( $n=68$ ) was composed of women who were first seen within the first 16 weeks of their pregnancies, and who remained free of periodontal disease throughout their pregnancies. Control Group I was set up as a comparison group under the supposition that, if it is really a risk factor for low-weight newborns, the incidence of this event in the Test Group should be greater than or equal to the incidence in the comparison group without this infection.

Control Group II $(n=112)$ was formed by women with periodontal disease who gave birth $<7$ days earlier, at the Women's Hospital of Feira de Santana. This hospital is a public institution that is responsible for approximately $50 \%$ of the live births among families of low socioeconomic level in the municipality. Control Group II was obtained from a previous evaluation carried out in 2004, by the same research team, and constituted the historical or external control. It was taken as a comparison group under the supposition that the occurrence of low birth weight should be greater in this group than in the test group if periodontal therapy is a protection factor for newborns with normal weight. The occurrence of low birth weight would be caused by the presence of periodontitis in Control Group II during the pregnancy, even though this condition was only diagnosed up to 7 days after delivery.

Women who were interested in entering the study were registered and given additional information about the research protocol. The study subjects were selected, periodontal therapy was administered, and support treatment was provided at the dental clinical of the State University of Feira de Santana, Bahia, Brazil. This study had previously been approved by the Research Ethics Committee of this university (Protocol No. 020/2002) and the Helsinki recommendations for conducting clinical research on human subjects were followed. Participants were enrolled after they had given their informed consent by signing a written declaration.

\section{Exclusion Criteria}

The exclusion criteria among the pregnant women evaluated, for all three groups, included presentation 
of fewer than six teeth, history of cardiovascular disease or any other systemic abnormality that would require antibiotic prophylaxis for the dental procedures, current use of corticosteroids, and presence of chronic kidney disease. In addition, women with a history of periodontal treatment within the last 6 months and women with multiple pregnancies (as assessed using ultrasonography) were excluded even though periodontal therapy continued to be administered until the end of the pregnancy. Lastly, women who did not complete the treatment or who did not return for reassessment, and those for whom the therapy was unsuccessful, were also excluded. The therapy was deemed unsuccessful in cases in which, at the time of reevaluation, the participant was again classified as presenting the disease, in accordance with the criteria for periodontitis described later.

\section{Measurements of the Periodontal Condition}

The study participants underwent a periodontal examination on all teeth present in their mouths, to assess their oral condition. All the clinical measurements, for all groups, were obtained by a single examiner (SSC) who was a general clinical dentist who had received prior training from an experienced periodontics specialist (ISGF). The intraexaminer concordance regarding the diagnosis of periodontitis was calculated by means of the $\kappa$ index, and a value of 0.823 was obtained for a difference of $\pm 1 \mathrm{~mm}$.

In the examination of periodontal condition, the sulcus/probing depth, gingival recession/hyperplasia, and bleeding on probing were measured for all teeth except for the third molars, and the values for clinical attachment loss that refers to the distance between the cemento-enamel junction and the base of the sulcus were obtained. These observations were made at six different locations on each tooth (distovestibular, mid-vestibular, mesio-vestibular, distolingual, mid-lingual, and mesio-lingual), with the aid of a Williams-type probe graduated in millimeters. The probing depth was recorded at each site as the distance from the gingival margin to the most apical extent of probe penetration. The measurements of the height of the gingival margin in relation to the cemento-enamel junction were considered to be positive values, because the gingival margin was found to be located apically to the cemento-enamel junction. In addition, the presence of gingival bleeding was observed for 10 seconds after removing the probe from the sulcus/pocket, after the probing depth procedure.

The periodontal examination also included recording the number of decayed teeth, missing teeth, and filled teeth, along with any other observed characteristic that was not within normal limits.

After gathering all the data, the patients were allocated to the Test Group or to Control Groups I or II.

\section{Diagnosis of Periodontal Disease}

The women were deemed to present a diagnosis of periodontitis if $\geq 4$ teeth had one or more sites with probing depth $\geq 4 \mathrm{~mm}$, with clinical attachment loss $\geq 3 \mathrm{~mm}$ at the same site and presence of bleeding on probing. ${ }^{23}$

\section{Recording of the Mother's Characteristics}

The pregnant women answered a questionnaire that prepared for this study. It was structured into two thematic sections. The first section covered identification and sociodemographic data, such as age at the start of the study, conjugal situation, education level, family income, and current occupation. The second section covered gestational history, including number of prenatal visits, nutritional condition (body mass index), existing pathologic conditions, urinary infection, other infectious diseases in the mother, number of previous pregnancies, previous abortions, use of medications, weight before the current pregnancy, weight at the time when previous children were born, and expectation regarding the type of delivery. Section two also asked about lifestyle habits, including smoking or alcohol consumption before or during the pregnancy (exposure to smoking was calculated by combining current and former smokers); and characteristics relating to oral health, including dental care and the type and frequency of tooth cleaning.

For the groups of women who were seen during their pregnancies (Test Group and Control Group I), this information was updated at subsequent interviews. Comparisons were made with the existing information on the pregnant women's attendance card or in the medical records filed in the municipal health care units relating to previous prenatal visits. The participants' body mass index was obtained every month. For this, an investigator who was a physical education professional (FPS) weighed the women on a digital balance. I On these occasions, this professional gave information to the women regarding the role of physical activity and the importance of adequate diet during pregnancy. For Control Group II, information was obtained from the medical records relating to the gestational period.

With regard to the quality of the prenatal examination, it is emphasized that it was theoretically the same for all of the participants, given that a standard attendance protocol for pregnant women is followed within the municipal health service. Because the women in the historical control group gave birth in the Women's Hospital, their prenatal attendance would have been provided within the municipal health service, which was set up by the Brazilian Ministry of Health in

I PCP10-SE, Hu-Friedy, Chicago, IL.

II Filizola S/A Industry, São Paulo, Brazil. 
1994, thus receiving the same care as mentioned previously. It is stressed that only the most prevalent characteristics among the pregnant women have been presented. Characteristics with few observations, or those that were infrequent in a pilot study that had previously been conducted on a sample of the same population, were excluded (e.g., type of delivery, frequency of the use of cigarettes and alcohol, asthma, and other illnesses).

\section{Periodontal Therapy}

The periodontal therapy for the pregnant women in the Test Group consisted of scaling and root planing, topical application of fluoride, and instruction and motivation toward oral hygiene. There was no use of antiseptics or antibiotics. The treatment was completed within 4 weeks and maintenance prophylaxis was implemented every 4 weeks thereafter, until the delivery. The latter consisted of scaling and root planing in areas with the presence of calculus, independent of the probing depth. Another complete periodontal examination was performed 30 days after the end of the therapy and after 6 and 8 months of gestation.

The pregnant women in Control Group I were monitored every 4 weeks during their pregnancies to maintain their periodontal health, with periodic prophylaxis, and to spot any changes in their periodontal condition. For both the Test Group and Control Group I, any lesions caused by caries and any other oral lesions were treated and any teeth indicated for exodontia were extracted. Periodontal treatment was made available to the women in Control Group II after the delivery, following the same procedures as applied to the Test Group.

\section{Evaluation of the Outcome}

The outcome evaluated was low birth weight, in accordance with World Health Organization definitions. ${ }^{24}$ Low birth weight was deemed to be present when the newborn's weight was $<2,500 \mathrm{~g}$, whereas a weight $\geq 2,500 \mathrm{~g}$ was considered normal. After the delivery, the recorded birth weight was collected from the birth certificate or from the newborn's card. It is emphasized that the protocol in the Ministry of Health's neonatal care manual was followed. This protocol was in use at the hospital institutions in which the deliveries took place. Thus, the mothers were classified as mothers of low-weight newborns, when the birth weight was $<2,500 \mathrm{~g}$; or mothers of normal-weight newborns, when the birth weight was $\geq 2,500 \mathrm{~g}$.

\section{Statistical Analysis}

The percentage distributions of all the covariables considered were analyzed: age, education level, conjugal situation, number of people living in the home, urinary infection, body mass index, diabetes, number of prenatal consultations, smoking habit, first pregnancy, and alcohol consumption. Depending on the indication, the $\chi^{2}$ or Fisher exact test was applied, with a significance level of $5 \%$, to evaluate any statistical differences between the frequencies of the abovementioned covariables, for the Test Group in relation to Control Groups I and II.

As a preparatory stage for modeling, stratified analysis was carried out to identify any potential confounders and effect modifiers. Multivariate analysis was carried out through unconditional logistic regression, using "backward" procedures. Odds ratio measurements were obtained and were converted into relative risk (RR) measurements by means of Poisson model. These were adjusted for confounding covariables and controlled for effect-modifying covariables, when applicable. The effect modifiers were identified as statistically significant results, with $\alpha$ equal to $5 \%$ in the likelihood ratio test. In the analysis on confounding, covariables that produced proportional differences of $>10 \%$ for the estimated RR were deemed to be confounders. ${ }^{25}$

The data analysis was performed using the STATA statistical software.\#

\section{RESULTS}

\section{Test Group Versus Control Group I}

The general percentage of low birth weight among the study sample was approximately $11 \%$. Sample characterization showed that the Test Group and Control Group I were comparable, given that there were no statistically significant differences $(P>0.05)$ for the covariables considered (Table 1). It is emphasized that none of the individuals in either group reported that they were smokers.

In the stratified analysis, there was neither evidence of effect modifiers nor evidence of confounding among the covariables investigated. Multivariate regression analysis on the Test Group and Control Group I also did not show any evidence of interactions, or any evidence of confounding in relation to the association under examination. Nevertheless, the covariables of age, education level, and smoking habit were kept in the model, in accordance with the theoretical suppositions, in which these characteristics are indicated as classic confounders. Considering the intervention design of the present study, it was decided to use the strategy of Poisson model to convert odds ratios into $R R$ values, thereby avoiding overestimation of the association. ${ }^{26}$ Thus, a crude association measurement of $R_{\text {crude }}=0.60$ and $95 \%$ confidence interval $(\mathrm{CI}), 0.34$ to 1.07 , was obtained, whereas the adjusted measurement was estimated to be of the

\# STATA, version 8.0, Stata Corp, College Station, TX. 
Table I.

\section{Sociodemographic and Lifestyle Characteristics of the Test Group and Control Group I}

\begin{tabular}{|c|c|c|c|c|c|}
\hline \multirow[b]{2}{*}{ Characteristics } & \multicolumn{2}{|c|}{$\begin{array}{l}\text { Test Group* } \\
(n=54)\end{array}$} & \multicolumn{2}{|c|}{$\begin{array}{l}\text { Control Group } I^{\dagger} \\
\qquad(n=68)\end{array}$} & \multirow[b]{2}{*}{$P$ Value } \\
\hline & $\mathrm{n}$ & $\%$ & $n$ & $\%$ & \\
\hline \multicolumn{6}{|l|}{ Age (years) } \\
\hline$\leq 18$ and $\geq 35$ & 15 & 27.8 & 10 & 14.7 & 0.08 \\
\hline 19 to 34 & 39 & 72.2 & 58 & 85.3 & \\
\hline \multicolumn{6}{|l|}{ Education level } \\
\hline 0 to 4 years & 8 & 14.8 & | | & 16.2 & 0.84 \\
\hline$\geq 5$ years & 46 & 85.2 & 57 & 83.8 & \\
\hline \multicolumn{6}{|l|}{ Conjugal situation } \\
\hline Single, divorced, or widowed & 20 & 37 & 25 & 36.8 & 0.97 \\
\hline Married or living together & 34 & 63 & 43 & 63.2 & \\
\hline \multicolumn{6}{|l|}{ Number of people in the home } \\
\hline$\geq 5$ & 16 & 29.6 & 15 & 22.1 & 0.34 \\
\hline$\leq 4$ & 38 & 70.4 & 53 & 77 & \\
\hline \multicolumn{6}{|l|}{ Urinary infection } \\
\hline Yes & | | & 20.4 & 14 & 20.9 & 0.98 \\
\hline No & 43 & 79.6 & 54 & 79.1 & \\
\hline \multicolumn{6}{|l|}{ Body mass index ${ }^{\S}$} \\
\hline$\geq 14$ and $<18.5 ; \geq 30$ and $\leq 37$ & 13 & 24.1 & 55 & 84.6 & 0.48 \\
\hline$\geq 18.5$ and $\leq 30$ & 41 & 75.9 & 10 & 15.4 & \\
\hline \multicolumn{6}{|l|}{ Diabetes } \\
\hline Yes & 2 & 3.7 & 0 & 0 & 0.11 \\
\hline No & 52 & 96.3 & 68 & 100 & \\
\hline \multicolumn{6}{|l|}{ Number of prenatal consultations } \\
\hline$<4$ & 6 & | 1.1 & 7 & 10.3 & 0.88 \\
\hline$\geq 4$ & 48 & 88.9 & 61 & 89.7 & \\
\hline \multicolumn{6}{|l|}{ Primiparous $\|$} \\
\hline Yes & 22 & 40.7 & 32 & 47.5 & 0.44 \\
\hline No & 32 & 59.3 & 35 & 52.5 & \\
\hline \multicolumn{6}{|l|}{ Alcohol consumption } \\
\hline Yes & 0 & 0 & । & 1.5 & 0.37 \\
\hline No & 54 & 100 & 67 & 98.5 & \\
\hline
\end{tabular}

* Test Group composed of women who were treated for periodontitis during their pregnancies.

$\dagger$ Control Group I composed of women without periodontitis during their pregnancies.

\# Statistical significance, $P \leq 0.05$

$\S$ Three samples from Control Group I were lost.

\| One sample from Control Group I was lost.
Test Group Versus Control Group II In comparing certain characteristics of the Test Group and Control Group II, it was observed that these were dissimilar regarding the distribution of the following covariables: education level, conjugal situation, number of people in the home, smoking habit, and alcohol consumption, for a statistical significance level of $5 \%$ (Table 3 ). It was also observed that the frequency of women at an age of risk for low birth weight $(\leq 18$ and $\geq 35)$ was higher in Control Group II (63.41\%) than in the Test Group (36.59\%).

In the stratified analysis, the findings did not present any evidence of effect modifiers. This analysis showed that education level was a candidate for confounding.

Multivariate regression analysis also did not present any evidence for interactions, although there was evidence for confounding with regard to education level. Once again, it was decided to keep age and smoking habit as covariables in the model, independent of the empirical results and in accordance with the theoretical suppositions that recognize both of these factors as classic confounders. Thus, a crude association measurement of $\mathrm{RR}_{\text {crude }}=2.13$ and 95\% CI, 1.30 to 3.48 , was obtained, whereas the adjusted measurement was of the order of $\mathrm{RR}_{\text {adjusted }}=1.92$ and $95 \% \mathrm{CI}, 1.15$ to 3.2 (Table 2). From this epidemiologic measurement, it was seen that the incidence of low birth weight in Control Group II was approximately twice the incidence seen in the Test Group. This result was statistically significant, for a $95 \% \mathrm{CI}$. order of $\mathrm{RR}_{\text {adjusted }}=0.57$ and $95 \% \mathrm{CI}, 0.31$ to 1.03 (Table 2). According to this epidemiologic measurement, it was found that the Test Group presented lower incidence of low birth weight than was observed in Control Group I. Furthermore, the precision measurement indicated that the incidence of low birth weight in the Test Group could vary and could take on a value at least equal to what was observed in Control Group I, for a $95 \% \mathrm{CI}$.

\section{DISCUSSION}

The main findings indicate that periodontal therapy may reduce the occurrence of low birth weight, although it has to be accepted that these results are preliminary. These results corroborate some of the existing investigations in the literature on this topic that involved the use of interventions. . $^{5,9,10,13,14,16-19}$ Among the studies that did not find an association are the investigations by Michalowicz et al., ${ }^{8,11}$ which 
Table 2.

RR and 95\% CI Measurements Obtained by Means of Logistic Regression for the Association Between Periodontal Therapy Among Pregnant Women and Low Birth Weight

\begin{tabular}{lcc}
\hline Association Measurements & $\begin{array}{c}\text { Test Group* Versus } \\
\text { Control Group }\left.\right|^{+}\end{array}$ & $\begin{array}{c}\text { Test Group* Versus } \\
\text { Control Group } \|^{*}\end{array}$ \\
\hline $\mathrm{RR}_{\text {crude }}$ & 0.6 & 2.13 \\
$95 \% \mathrm{Cl}$ & 0.34 to 1.07 & 1.30 to 3.48 \\
$\mathrm{RR}$ adjusted & $0.57 \S$ & $1.92^{\S}$ \\
$95 \% \mathrm{Cl}$ & 0.31 to 1.03 & 1.15 to 3.2 \\
\hline
\end{tabular}

* Test Group composed of women who were treated for periodontitis during their pregnancies. † Control Group I composed of women without periodontitis during their pregnancies.

$\ddagger$ Control Group II composed of women with periodontitis diagnosed during the immediate postpartum period

$\S$ Age, education level, and smoking habit.

were published in 2006 and 2009. It is worth stressing that the criterion for exposure measurement used in those studies was shaky, given that they used attachment loss $>2 \mathrm{~mm}$ as the diagnosis for periodontitis. Such situations may represent the beginnings of gingival recession, for example because of mechanical trauma. Use of this criterion would contribute toward changing the value of the final association. ${ }^{23}$

There is an understanding that studies in the literature have not established whether periodontal treatment during pregnancy has any real benefit with regard to preventing undesirable gestational outcomes. ${ }^{11,16}$ Nevertheless, some research ethics committees (institutional review boards), such as in Brazil, have not agreed with the criterion of randomization and selection of groups for treatment. This was the case with the final opinion from the ethics committee regarding the project for the present study. Thus, methodologic resources were sought from the field of epidemiology, to minimize the effects of non-randomization, through the use of multiple controls, focusing on a historical control in which the comparison subjects had not undergone periodontal therapy at any given time in the past. Moreover, few papers on this topic, using this investigation strategy, were found in the literature.

Two working hypotheses were tested. In hypothesis one, investigated through the Test Group and Control Group I, it was suggested that the risk of low birth weight in the Test Group would be less than or equal to what was observed in Control Group I. In hypothesis two, it was suggested that the frequency of low birth weight in the Test Group would be lower than in Control Group II.
Through the study hypothesis, a group of 54 pregnant women who underwent periodontal treatment during pregnancy was investigated. These women were compared with another group of 112 women in the immediate postpartum period who had not had previous therapy. It was ensured that all of these subjects came from the same population of pregnant women who were attending one of the public health care clinics in the municipality of Feira de Santana. These public clinics do not provide periodontal care during pregnancy, despite acceptance of the hypothesis that, during this phase of life, women present greater vulnerability because of pregnancyrelated hormone changes. ${ }^{27}$ It was observed that low-weight births occurred twice as frequently among the untreated women than among the women in the test group, who had undergone periodontal therapy during their pregnancies.

This alternative methodology has attracted many criticisms because of the existence of sources of nonequivalence between the test and control groups, dispersed over time, thereby giving rise to violation comparability. ${ }^{28}$ On the other hand, the use of historical controls is not rare, especially in health and educational program evaluations and in clinical fields (e.g., surgery). ${ }^{29}$

In the present study, it was found that the Test Group and Control Group II were dissimilar regarding the distribution of some covariables, for example education level, conjugal situation, number of people in the home, smoking habit, and alcohol consumption. In situations like these, indicating lack of comparability between the groups, appropriate use of analytical methods, like the ones used in this investigation, is required to control for unequally distributed baseline factors. Such factors could have been eliminated through randomization.

Thus, although only the education level was considered to be a confounder following the empirical analysis, both age and smoking habit were incorporated into the final model from the analysis, on the grounds of a priori knowledge, ${ }^{30,31}$ to minimize possible distortions of the association measurement investigated. Other variables, such as the number of people in the home, did not form part of the model because they were considered to be proxy variables for the education level, and characteristic of the socioeconomic condition of the groups studied. In this way, to avoid over-adjustment, particularly because of variables that measure related events, and consequently to eliminate 
Table 3.

\section{Sociodemographic and Lifestyle Characteristics of the Test Group and Control Group II}

\begin{tabular}{|c|c|c|c|c|c|}
\hline \multirow[b]{2}{*}{ Characteristics } & \multicolumn{2}{|c|}{$\begin{array}{l}\text { Test Group* } \\
(n=54)\end{array}$} & \multicolumn{2}{|c|}{$\begin{array}{l}\text { Control Group } \|^{\dagger} \\
\quad(n=112)\end{array}$} & \multirow[b]{2}{*}{$P$ Value ${ }^{\ddagger}$} \\
\hline & $\mathrm{n}$ & $\%$ & $n$ & $\%$ & \\
\hline \multicolumn{6}{|l|}{ Age (years) } \\
\hline$\leq 18$ and $\geq 35$ & 15 & 27.8 & 26 & 23.2 & 0.52 \\
\hline 19 to 34 & 39 & 72.2 & 86 & 76.8 & \\
\hline \multicolumn{6}{|l|}{ Education level } \\
\hline 0 to 4 years & 6 & 11.1 & 47 & 42 & 0.00 \\
\hline$\geq 5$ years & 48 & 88.9 & 65 & 58 & \\
\hline \multicolumn{6}{|l|}{ Conjugal situation§ } \\
\hline Single, divorced, or widowed & 20 & 37 & 27 & 24.3 & 0.09 \\
\hline Married or living together & 34 & 63 & 84 & 75.7 & \\
\hline \multicolumn{6}{|l|}{ Number of people in the home } \\
\hline$\geq 5$ & 17 & 31.5 & 64 & 57.1 & 0.00 \\
\hline$\leq 4$ & 37 & 68.5 & 48 & 42.9 & \\
\hline \multicolumn{6}{|l|}{ Urinary infection } \\
\hline Yes & 11 & 20.4 & 29 & 25.9 & 0.44 \\
\hline No & 43 & 79.6 & 83 & 74.1 & \\
\hline \multicolumn{6}{|l|}{ Body mass index } \\
\hline$\geq 14$ and $<18.5 ; \geq 30$ and $\leq 37$ & 13 & 24.1 & 23 & 20.5 & 0.06 \\
\hline$\geq 18.5$ and $\leq 30$ & 41 & 75.9 & 89 & 79.5 & \\
\hline \multicolumn{6}{|l|}{ Diabetes ${ }^{\S}$} \\
\hline Yes & 2 & 3.7 & 0 & 0 & 0.11 \\
\hline No & 52 & 96.3 & 111 & 100 & \\
\hline \multicolumn{6}{|l|}{ Number of prenatal consultations } \\
\hline$<4$ & 46 & 85.2 & 71 & 63.4 & 0.04 \\
\hline$\geq 4$ & 8 & 14.8 & 41 & 36.6 & \\
\hline \multicolumn{6}{|l|}{ Smoking habit } \\
\hline Yes & 0 & 0 & 8 & 7.1 & 0.04 \\
\hline No & 54 & 100 & 104 & 92.9 & \\
\hline \multicolumn{6}{|l|}{ Primiparous } \\
\hline Yes & 22 & 40.7 & 34 & 30.4 & 0.18 \\
\hline No & 32 & 59.3 & 78 & 69.6 & \\
\hline \multicolumn{6}{|l|}{ Alcohol consumption } \\
\hline Yes & I & 1.8 & 16 & 14.3 & 0.01 \\
\hline No & 53 & 98.2 & 96 & 85.7 & \\
\hline
\end{tabular}

* Test Group composed of women who were treated for periodontitis during their pregnancies.

$\dagger$ Control Group II composed of women with periodontitis that were diagnosed during the immediate postpartum period.

\# Statistical significance, $P \leq 0.05$

$\S$ One sample from Control Group II was lost.

bias from the effect measurements, the number of covariables in the model was limited to those mentioned previously. This also took into account the analytical supposition of a minimum relationship of 10 response events for each independent variable in the model analyzed. ${ }^{32}$ However, it is important to emphasize that other characteristics of the sample that influenced the association also may not have been measured for a variety of reasons. This would constitute a source of residual confounding. ${ }^{33,34}$

To avoid compromising the methodologic quality, given that for ethical reasons a classic intervention study in which the control group would be formed by women with periodontitis could not be conducted, and given the question of nonequivalence between the groups (absence of randomization), one complementary alternative resource is to use another comparison group. This would also increase the base of evidence for testing the hypothesis of an association. In the present investigation, Control Group I was formed from pregnant women without periodontitis. This control group and the test group presented equivalence with regard to the distribution of the covariables investigated.

In this second hypothesis, it was expected that the incidence of low birth weight in the treated group would be similar to what was observed in the control group without disease, if the treatment were effective. The findings from the present study confirmed this hypothesis and strengthened the evidence for the existence of this association. They also complemented the results from the other groups presented previously.

Another point that can be criticized concerns the time of exposure in relation to the outcome in Control Group II (historical control), because the periodontitis was diagnosed only during the postpartum period. To settle any doubt regarding the pres ence of periodontal disease during pregnancy in this group, the diagnosis was made within 7 days of the delivery. Robust diagnostic criteria with good specificity were used ${ }^{23}$ with the aim of avoiding misclassification of the periodontal status. These criteria consisted of three essential conditions that were present at the 
same site, in $\geq 4$ teeth: 1 ) probing depth $>4 \mathrm{~mm}$; 2) clinical attachment loss $>3 \mathrm{~mm}$; and 3 ) bleeding on probing. This choice of criteria was also based on the fact that periodontal disease progresses in stages $^{35}$ and that the clinical characteristics of gingival inflammation appear by the 21 st day after complete cessation of all means of oral hygiene. After this period, destruction of the periodontal support may occur, although the exact time when this loss (periodontitis) occurs is not known. Thus, it is certain that these women's periodontal infection was present during their pregnancies because the development of tissue alterations for periodontitis to occur requires much more than just 1 week.

It is also important to emphasize that, although no calculation was made to determine the sample size $(n=234)$, this was close to the number of participants $(\mathrm{n}=302)$, in the study by Cruz et al. ${ }^{36}$ The latter had characteristics similar to those of the present investigation, given that the two studies were generated from the same population. On the other hand, it is accepted that the methodologic strategy presented here needs to be applied to studies that have larger samples.

The ethical impositions inherent to experimental studies, together with the great complexity of such studies, mean that greater time is required and greater quantities of human resources with higher qualifications are needed. Moreover, such studies are very expensive to conduct.

\section{CONCLUSIONS}

The findings from the present investigation show that pregnant women suffering from periodontitis who were successfully treated during pregnancy had the same rate of occurrence of low birth weight as shown by pregnant women without periodontitis. The small number of intervention studies on the present topic makes it clear that the methodologic strategy (experimental design with multiple controls, with emphasis on a historical control group) used in the present investigation is of importance as a further alternative that can be used in research on the influence of periodontal therapy on low-weight newborns.

\section{ACKNOWLEDGMENTS}

The authors thank everyone who formed part of the human resources for achieving this study. We thank the women who formed part of the sample for this study, for their contribution toward the process of investigating this topic. We also thank the Research Support Foundation of the State of Bahia (FAPESB), Salvador, Bahia, Brazil, and Feira de Santana State University (UEFS), Feira de Santana, Bahia, Brazil, for financial support for this research. The authors report no conflicts of interest related to this study.

\section{REFERENCES}

1. Vettore MV, Lamarca Gde A, Leão AT, Thomaz FB, Sheiham A, Leal Mdo C. Periodontal infection and adverse pregnancy outcomes: A systematic review of epidemiological studies. Cad Saude Publica 2006;22: 2041-2053.

2. Khader Y, Al-shishani L, Obeidat B, et al. Maternal periodontal status and preterm low birth weight delivery: A case-control study. Arch Gynecol Obstet 2009;279:165-169.

3. Durand R, Gunselman EL, Hodges JS, Diangelis AJ, Michalowicz BS. A pilot study of the association between cariogenic oral bacteria and preterm birth. Oral Dis 2009;15:400-406.

4. Cruz SS, Costa Mda C, Gomes-Filho IS, et al. Contribution of periodontal disease in pregnant women as a risk factor for low birth weight. Community Dent Oral Epidemiol 2009;37:527-533.

5. López NJ, Smith PC, Gutierrez J. Periodontal therapy may reduce the risk of preterm low birth weight in women with periodontal disease: A randomized controlled trial. J Periodontol 2002;73:911-924.

6. Jeffcoat MK, Hauth JC, Geurs NC, et al. Periodontal disease and preterm birth: Results of a pilot intervention study. J Periodontol 2003;74:1214-1218.

7. López NJ, Da Silva I, Ipinza J, Gutiérrez J. Periodontal therapy reduces the rate of preterm low birth weight in women with pregnancy-associated gingivitis. J Periodontol 2005;76(Suppl. 11):2144-2153.

8. Michalowicz BS, Hodges JS, DiAngelis AJ, et al; Treatment of periodontal disease and the risk of preterm birth. N Engl J Med 2006;355:1885-1894.

9. Gazolla CM, Ribeiro A, Moysés MR, Oliveira LAN, Pereira LJ, Sallum AW. Evaluation of the incidence of preterm low birth weight in patients undergoing periodontal therapy. J Periodontol 2007;78:842-848.

10. Tarannum F, Faizuddin M. Effect of periodontal therapy on pregnancy outcome in women affected by periodontitis. J Periodontol 2007;78:2095-2103.

11. Michalowicz BS, Hodges JS, Novak MJ, et al. Change in periodontitis during pregnancy and the risk of preterm birth and low birthweight. J Clin Periodontol 2009;36:308-314.

12. Offenbacher S, Beck JD, Jared HL, et al; Maternal Oral Therapy to Reduce Obstetric Risk (MOTOR) Investigators. Effects of periodontal therapy on rate of preterm delivery: A randomized controlled trial. Obstet Gynecol 2009;114:551-559.

13. Oliveira AM, de Oliveira PA, Cota LO, Magalhães CS, Moreira AN, Costa FO. Periodontal therapy and risk for adverse pregnancy outcomes. Clin Oral Investig 2010; May 22 [Epub ahead of print].

14. Cruz SS, Costa Mda C, Gomes-Filho IS, et al. Periodontal therapy for pregnant women and cases of low birthweight: An intervention study. Pediatr Int 2010; $52: 57-64$

15. Gluud LL. Bias in clinical intervention research. Am J Epidemiol 2006;163:493-501.

16. Macones GA, Parry S, Nelson DB et al. Treatment of localized periodontal disease in pregnancy does not reduce the occurrence of preterm birth: Results from the Periodontal Infections and Prematurity Study (PIPS). Am J Obstet Gynecol 2010;202:147.1-147.8.

17. Radnai M, Pál A, Novák T, Urbán E, Eller J, Gorzó I. Benefits of periodontal therapy when preterm birth threatens. J Dent Res 2009;88:280-284. 
18. Novák T, Radnai M, Gorzó I, et al. Prevention of preterm delivery with periodontal treatment. Fetal Diagn Ther 2009;25:230-233.

19. Deppe H, Hohlweg-Majert B, Hölzle F, Schneider KT, Wagenpfeil S. Pilot study for periodontal treatment and pregnancy outcome: A clinical prospective study. Quintessence Int 2010;41:e101-e110.

20. Wimmer G, Pihlstrom BL. A critical assessment of adverse pregnancy outcome and periodontal disease. J Clin Periodontol 2008;35(Suppl. 8):380-397.

21. Coutinho SB, Lima MC, Ashworth A, Lira PI. The impact of training based on the Baby-Friendly Hospital Initiative on breastfeeding practices in the Northeast of Brazil (in Portuguese). J Pediatr 2005;81:471-477.

22. Morris CR, Harvey IM, Stebbings WS, Speakman CT, Kennedy HJ, Hart AR. Do calcium channel blockers and antimuscarinics protect against perforated colonic diverticular disease? A case control study. Gut 2003; 52:1734-1737.

23. Gomes-Filho IS, Cruz SS, Rezende EJ, et al. Exposure measurement in the association between periodontal disease and prematurity/low birth weight. $J$ Clin Periodontol 2007;34:957-963.

24. World Health Organization. ICD 10 - International Statistical Classification of Diseases and Related Health Problems, 2nd edition. Geneva, Switzerland: World Health Organization; 2005.

25. Rothman KJ, Greenland S. Modern Epidemiology. Philadelphia: Lippincott Williams \& Wilkins; 1986: 285-310.

26. Barros AJ, Hirakata VN. Alternatives for logistic regression in cross-sectional studies: An empirical comparison of models that directly estimate the prevalence ratio. BMC Med Res Methodol 2003; 3:21.

27. Zachariasen RD. The effect of elevated ovarian hormones on periodontal health: Oral contraceptives and pregnancy. Women Health 1993;20:21-30.
28. Sacks H, Chalmers TC, Smith H Jr. Randomized versus historical controls for clinical trials. Am J Med 1982;72:233-240.

29. Silverman HJ, Miller FG. Control group selection in critical care randomized controlled trials evaluating interventional strategies: An ethical assessment. Crit Care Med 2004;32:852-857.

30. González YM, De Nardin A, Grossi SG, Machtei EE, Genco RJ, De Nardin E. Serum cotinine levels, smoking, and periodontal attachment loss. J Dent Res 1996;75:796-802.

31. Tomar SL, Asma S. Smoking-attributable periodontitis in the United States: Findings from NHANES III. $J$ Periodontol 2000;71:743-751.

32. Concato J, Feinstein AR, Holford TR. The risk of determining risk with multivariable models. Ann Intern Med 1993;118:201-210.

33. Kunz R, Oxman AD. The unpredictability paradox: Review of empirical comparisons of randomized and non-randomized clinical trials. BMJ 1998;317:11851190.

34. Milne E, Johnston R, Cross D, Giles-Corti B, English DR. Effect of a school-based sun-protection intervention on the development of melanocytic nevi in children. Am J Epidemiol 2002;155:739-745.

35. Löe H, Theilade E, Jensen SB. Experimental gingivitis in man. J Periodontol 1965;36:177-187.

36. Cruz SS, Costa Mda C, Gomes Filho IS, Vianna MI, Santos CT. Maternal periodontal disease as a factor associated with low birth weight [in Portuguese]. Rev Saude Publica 2005;39:782-787.

Correspondence: Dr. Isaac Suzart Gomes-Filho, Av. Getúlio Vargas, 379, Centro, Feira de Santana, Bahia 44.025-010, Brazil. Fax: 55-75-3623-0661; e-mail: isuzart@gmail.com.

Submitted January 25, 2010; accepted for publication June 25, 2010. 